\title{
The complications associated with guidewire use in spine surgeries involving pedicle screw placement: A comprehensive literature review
}

\author{
John B. Pracyk ${ }^{1}$, Nicole Ferko*2, Adrian P. Turner ${ }^{2}$, Sara N. Root ${ }^{1}$, Heather Cannon ${ }^{1}$, Michael Erb ${ }^{1}$, Ann M. Menzie ${ }^{1}$ \\ ${ }^{1}$ DePuy Synthes Spine, United States \\ ${ }^{2}$ Cornerstone Research Group, Canada
}

Received: September 21, 2018

Accepted: November 28, 2018

Online Published: December 7, 2018

DOI: $10.5430 /$ css.v5n1p14

URL: https://doi.org/10.5430/css.v5n1p14

\begin{abstract}
Guidewires (Kirschner or "K" wires) are often required during minimally invasive spine surgery to facilitate percutaneous pedicle screw placement. The use of guidewires involves a multi-step process that carries the risk of complications and their associated consequences. To date, the reporting of such information has been limited, and the literature has not been thoroughly evaluated. The objective of this study was to conduct a narrative review and assess the burden associated with guidewire use in spine surgeries. Databases searched included PubMed and Embase between the years of 1988 and 2017. In addition to databases, recent data from relevant trade journals were hand-searched. Inclusion criteria were broad to avoid potential exclusion of relevant publications. In total, 31 articles were included. This review found that the risk of complications associated with guidewire use in spine procedures ranged from $0.4 \%$ to $14.8 \%$. Complication types included guidewire fracture, cerebrospinal fluid leakage, post-operative ileus, infection, and other spinal hardware failure (e.g., pedicle screw pull-out). Causes of complications typically included breakage and migration of the guidewire (metal fatigue), inexperience with guidewire use, or lack of tactile or visual feedback. Specific surgery types or patient populations may be more susceptible to guidewire-related complications (e.g., L5-S1 level operations). Complications associated with guidewire use may also lead to healthcare resource utilization, including additional operating time, radiation exposure, and re-operations. Solutions to help minimize the risk of such complications and associated consequences are required.
\end{abstract}

Key Words: K-wire, Minimally invasive, Pedicle screws, Spine, Complications, Literature review

\section{INTRODUCTION}

Guidewires are often used in orthopedic surgeries; they are available in a variety of shapes and sizes and are driven through bone to help guide a surgeon as they insert subsequent surgical hardware. ${ }^{[1]}$ In spine procedures, guidewires-often referred to as K-wires-are commonly used in minimally invasive surgery (MIS), such as transforaminal lumbar interbody fusion (TLIF) and stabilization in spinal trauma. ${ }^{[2]}$ In MIS fusion, the use of guidewires is a multi-step process which involves fluoroscopy for visualization of several instruments (e.g., Jamshidi needle, tap, etc.) to facilitate a path for proper pedicle screw insertion. Fusion procedures remain an important method of spinal stabilization in the management of deformity, degenerative disc disease, neoplasms, trauma, and other conditions that affect the spinal column. ${ }^{[1]}$

*Correspondence: Nicole Ferko; Email: nferko@ cornerstone-research.com; Address: Cornerstone Research Group, Canada. 
Although guidewires help with ensuring proper pedicle screw placement, they may present challenges to both patient and surgeon. It has been previously reported that between $10 \%$ and $20 \%$ of patients who undergo a surgical spinal procedure suffer adverse events or complications; however, there have been conflicting data on causative factors. ${ }^{[3]}$ In particular, the overall risk of complications and consequences associated with guidewire use in MIS spinal procedures remains poorly characterized. ${ }^{[4]}$ Given the recent initiatives focused on improving patient outcomes and reducing costs, minimizing surgical complications is of high interest to healthcare stakeholders. As use of guidewires may be associated with complications, radiation exposure through fluoroscopy, and associated healthcare resource use, the objective of this literature review was to evaluate and characterize such implications and identify areas for improvement.

\section{MethodS}

A PubMed, Embase, and Google search, which focused on guidewire use in spinal surgeries, was undertaken to inform this narrative review. The term "guidewire" was found to be more commonly referred to as Kirschner wire or K-wire, in spinal literature. A search strategy was then developed by the authors (which also included input from an information specialist) and searched in PubMed and Embase on November 7, 2017. The following limits were placed on the search: publication dates: 1988 to 2017 (in Embase) and species: human. Language limits were not applied to the search. Search terms reflected a combination of medical subject headings (MeSH) and keywords (see Table 1). In addition to the Google, PubMed, and an Embase search, the authors also identified two relevant trade journals, with full-text available online, that were subsequently hand-searched for relevant material. Specifically, the trade journals "OR Manager" and "Outpatient Surgery Magazine" were reviewed from the past 3 years (2015-2017). Articles were also identified through reference lists of relevant publications.

The information specialist performed the initial search. The abstracts of the publications from the search were then reviewed for potential inclusion by two authors (APT and NF). The second author (NF) helped to determine the relevance for abstract inclusion once the first author had conducted screening. The full-text article was reviewed in detail for those studies identified as possibly relevant. As part of full-text review, no restrictions were placed on articles that reported complications or other challenges associated with guidewire use in spine procedures. Formal quality assessment using standardized rating scales was not completed for this review given its non-systematic nature and the fact that various clinical study designs, including case reports, were included in this review.

Table 1. Literature search strategy

\begin{tabular}{|c|c|}
\hline & Keywords \\
\hline 1 & $\begin{array}{l}\text { ("K-wire" or "K-wires" or "K-wireless" or "K-wire-less" or Kirshner [tw] or GW or GWs or GWless or GW-less or Bone } \\
\text { Wires [MeSH]) AND }\end{array}$ \\
\hline 2 & 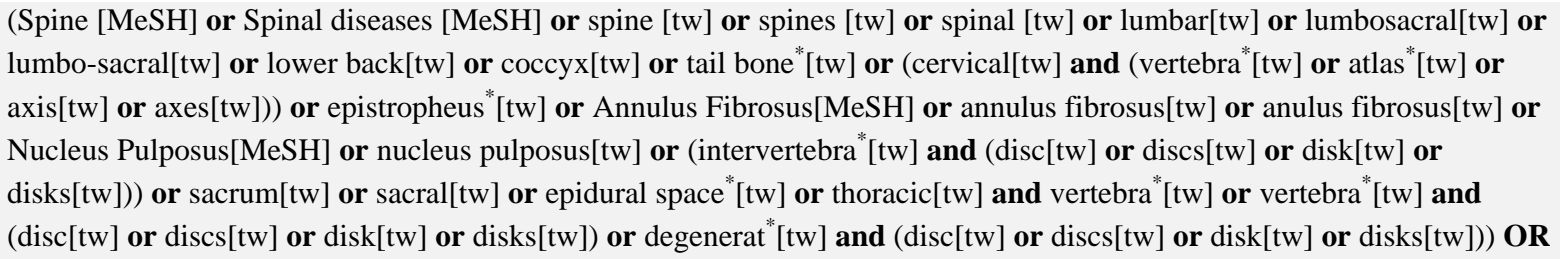 \\
\hline 3 & $\begin{array}{l}\text { Diskectomy }[\mathrm{MeSH}] \text { or } \operatorname{diskectom}^{*}[\mathrm{tw}] \text { or } \operatorname{discectom}^{*}[\mathrm{tw}] \text { or laminectom }{ }^{*}[\mathrm{tw}] \text { or laminotom }{ }^{*}[\mathrm{tw}] \text { or Spinal Fusion }[\mathrm{MeSH}] \\
\text { or spondylodes }[\mathrm{tw}] \text { or spondylosyndes }{ }^{*}[\mathrm{tw}] \text { or vertebra }{ }^{*}[\mathrm{tw}] \text { and fusion }[\mathrm{tw}] \text { and }\left(\operatorname{surger}^{*}[\mathrm{tw}] \text { or surgical }{ }^{*}[\mathrm{tw}] \text { or }\right. \\
\left.\left.\text { operate }[\mathrm{tw}] \text { or operated }[\mathrm{tw}] \text { or operates }[\mathrm{tw}] \text { or operating }[\mathrm{tw}] \text { or operation }[\mathrm{tw}] \text { or operations }[\mathrm{tw}] \text { or procedur }{ }^{*}[\mathrm{tw}]\right)\right) \text { OR }\end{array}$ \\
\hline 4 & (Osteoporosis[MeSH] or osteoporo $[\mathrm{tw}]$ or bone loss $\left.{ }^{*}[\mathrm{tw}]\right)$ OR \\
\hline 5 & (Bone Screws $[\mathrm{MeSH}]$ or bone screw ${ }^{*}[\mathrm{tw}]$ or jamshidi needle ${ }^{*}[\mathrm{tw}]$ or pedicle screw ${ }^{*}[\mathrm{tw}]$ ) \\
\hline
\end{tabular}

\section{RESUlts}

The literature search resulted in 2,058 abstracts for review. In brief, most abstracts were excluded because they were not related to the spine (e.g., wrist, clavicle, etc.) or because the guidewire discussed was not relevant (e.g., gynecological, cardiovascular, etc.). Of the abstracts, 45 articles were selected for full-text review, and 31 articles were determined

Published by Sciedu Press to be relevant for full data abstraction. All of these were reported in the English language. Articles included were those that reported research relating to any of the following with respect to guidewire use in spine surgery: complications, consequences of complications, fluoroscopy, radiation exposure, healthcare resource use (e.g., operative time), procedure or process (in)efficiencies, and utilization of guidewire-less 
techniques.

\subsection{Complication types}

Many of the studies reported on complications associated with MIS of the spine (i.e., mainly spinal fusions). A detailed review of the 31 relevant studies, including all clinical study design types, yielded several types of complications that have been noted to be causally linked or possibly related to guidewire use:

- Spinal hardware failure (e.g., pedicle screw pull-out, K-wire fracture/breakage $)^{[5-13]}$

- Blood loss ${ }^{[7,9]}$

- CSF leak ${ }^{[7,14]}$

- Infection (e.g., osteomyelitis, septicemia, etc. $)^{[14-17]}$

- Bone fracture ${ }^{[18]}$

- Paraplegia ${ }^{[19]}$

- Injury to the bladder or abdominal viscera ${ }^{[17]}$

- Retroperitoneal hematoma ${ }^{[4]}$

- Post-operative ileus ${ }^{[2,4,7]}$

- Hematoma ${ }^{[4]}$

- Cardiac tamponade ${ }^{[20]}$

- Death ${ }^{[20]}$

- Other neural or vascular complications ${ }^{[17,21,22]}$

Additionally, greater blood loss has also been shown to be associated with guidewire use, when compared to other spinal surgical techniques (i.e., awl-staple method). ${ }^{[9]}$ Furthermore, blood loss has been demonstrated to be high in certain cases of guidewire complications; for example, one case reported an estimated blood loss of 2 liters. ${ }^{[7]}$

\subsection{Complication risk}

The rate and type of complications associated with guidewire use in spine procedures is summarized in Table 2. Only studies with sample sizes of greater than 10 patients were reported in Table 2 for this outcome (i.e., excludes case reports). The review found that complication risk ranged from $0.4 \%$ to $14.8 \%$ across all studies. Most studies reported complication rates that were lower than 5\%; many of which were retrospective in study design. Interestingly, a published systematic review of thoracolumbar spine procedures revealed that prospective studies demonstrated twice the likelihood of complications compared with retrospective studies. ${ }^{[3]}$ This may be in part due to the fact that outcome measures can often be more precisely defined, captured, and evaluated within the context of a prospectively designed study.

\subsection{Causes and consequences of complications}

The literature revealed several causes of guidewire associated complications. A guidewire breach of a nearby anatomical structure is a common reason for a complication. The depth to which there is a guidewire breach has an impact on the severity of the complication (see Table 3$)^{[4]}$ as well as resource use associated with the breach. For example, a moderate/major breach could result in additional laboratory tests (e.g., blood gases, hemoglobin, etc.), diagnostic tests (e.g., computer tomography [CT] scan, etc.), general/vascular surgery consult, or antibiotic treatment. ${ }^{[4]}$

The literature reports that guidewire complications are often related to breakage and migration of the guidewire, which can damage surrounding neural and vascular structures or visceral organs (as reported in Table 3). ${ }^{[4,23]}$ Additional reasons cited for guidewire-associated complications are metal fatigue of the guidewire, surgeon inexperience with guidewires, and lack of tactile or visual feedback during MIS spinal procedures. ${ }^{[13,23]}$

Table 2. Summary of guidewire-associated complication rates in spinal surgeries

\begin{tabular}{llllll}
\hline Authors \& Years & Study Design & Procedure Details & Sample Size $^{*}$ & Guidewire Complication Type & Complication Rate (\%) \\
\hline Shim et al., 2005 & Prospective & ALIF & 20 & Articular process fracture & 5.0 \\
Ringstrom et al., 2007 & Retrospective & PSP & 25 & Osteomyelitis with septicemia & 0.98 \\
Agrawal \& Sucato, 2008 & Retrospective & AF & 27 & Screw pullout & 14.8 \\
Hao et al., 2008 & Retrospective & PSP & 103 & CSF leak, infection & 1.94 \\
Lau et al., 2013 & Retrospective & MIS-TLIF & 127 & K-wire fracture & 1.28 \\
Mobbs et al., 2014 & Retrospective & PSP & 525 & Post-operative ileus & 0.4 \\
Scheer et al., 2014 & Prospective & MIS-TLIF & 513 & K-wire fracture, CSF leak, ileus & 1.2 \\
Wong et al., 2014 & Prospective & MIS-TLIF & 144 & Post-operative ileus & 0.69 \\
Guiroy et al., 2017 & Prospective & MIS-TLIF & 38 & K-wire fracture & 2.6 \\
\hline
\end{tabular}

Note. MIS-TLIF: minimally invasive transforaminal lumbar interbody fusion; CSF: cerebrospinal fluid; ALIF: Anterior lumbar interbody fusion; AF: anterior spinal fusion;

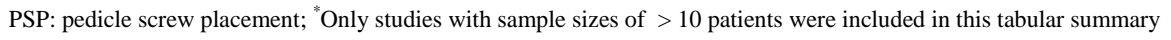


Table 3. Depth of guidewire and potential complications during percutaneous pedicle screw insertion ${ }^{[4]}$

\begin{tabular}{|c|c|c|}
\hline Depth & Structures at Risk & Potential Complications \\
\hline$<5 \mathrm{~mm}$ & Sympathetic plexus & $\begin{array}{l}\text { Sympathetic lesions (ejaculatory dysfunction, altered temperature perception, and } \\
\text { perspiration in lower extremities) }\end{array}$ \\
\hline $5-25 \mathrm{~mm}$ & Above structures, major vessels & Vascular injury, aneurysm or pseudo aneurysm, retroperitoneal bleed \\
\hline$>25 \mathrm{~mm}$ & Above structures, bowel & Bowel perforation (ileus, peritonitis, sepsis) \\
\hline
\end{tabular}

Note. Table originally cited in Mobbs \& Raley, 2014 ${ }^{[4]}$

Specific operations may be more likely to result in guidewire breakage. Surgeries involving L5-S1 level operation often need a larger entry angle to successfully insert the guidewire. At such an angle, the guidewire is more likely to be broken and subsequently leave the patient at a higher risk of developing a complication. ${ }^{[13]}$ Caution should be taken throughout the surgery, specifically when instruments are being moved over the guidewires; if screws/taps are inserted at a different angle from the guidewire, the guidewire may kink and instruments/implants may become caught on the guidewire which could inadvertently displace the guidewire and breach internal arteries or visceral structures. ${ }^{[17]}$ Furthermore, some studies have observed that guidewire complications may be more prevalent in specific patient types and surgeries. For example, both osteoporotic and obese patients have been reported to be at increased risk of guidewire-associated complications. ${ }^{[10,17,19,23]}$ In osteoporotic patients, guidewires can more easily advance through the vertebral body, causing visceral or vascular injury. ${ }^{[23]}$ As such, increased caution is warranted when using guidewires in those specific patient populations.

Complications associated with guidewire use can result in downstream effects, that can negatively affect patient outcomes and healthcare resource use. Such consequences can include patient morbidity, additional operative time, increased radiation exposure, and surgeon frustration; ${ }^{[23]}$ some of these consequences are expanded upon in later sections. Although guidewire complication rates are reportedly low, there is concern that this may be an underestimation of the true complication incidence, especially those reported in retrospective analyses. ${ }^{[3]}$ Overall, guidewire complications are concerning, and the proper precautions should be taken to mitigate the risks, which if ignored may result in serious and significant consequences.

\subsection{Procedural inefficiencies}

Guidewire use, and its associated complications, may lead to additional operating time, surgical revisions, and intraoperative conversion from MIS to open procedures. For example, fractured or broken guidewires can increase operating time and risk of conversion to open procedures. ${ }^{[6,8,13]}$ In contrast to experienced surgeons performing an MIS pro- cedure, a less experienced surgeon may need to transition to an open procedure to properly visualize and retrieve a fractured or broken guidewire. ${ }^{[6]}$ Guidewire use can also result in the need for surgical revisions to correct misaligned pedicle screws if the screw did not follow the path formed by the guidewire. Santos and colleagues showed that screws inserted percutaneously had a significantly higher rate of revision as compared with those inserted using an open technique, when only guidewire revisions were included in the analysis $(7.5 \%$ vs. $2.7 \%, p=.0004){ }^{[24]}$

With increased operative times, the need for more anesthesia is probable. Evidence demonstrates a greater risk of medical complications with increased anesthesia use, specifically in pedicle screw-based spinal procedures. ${ }^{[16]}$ Specifically, longer anesthesia times are associated with an increased incidence of intraoperative technical errors, infections, blood loss, and postoperative mortality. ${ }^{[16]}$ With conversion to open surgery, not only is increased anesthesia possible, but the conversion itself subjects patients to the risks of complications typically associated with open spinal surgery, such as increased infection, hematoma or deep vein thrombosis risk, increased post-operative pain, and longer length of stay. ${ }^{[2]}$

\subsection{Radiation exposure}

Fluoroscopy is necessary with guidewire use in MIS spine procedures to visualize spinal and anatomical structures. Compared with open procedures, MIS is associated with a 2.5 -fold increase in radiation $(1.90 \mathrm{mSv}$ vs. $0.75 \mathrm{mSv}$, $p<.01){ }^{[2]}$ With increased fluoroscopy use, there becomes a greater concern for the safety of the surgeon, operating room staff, and the patient. It is well-known that excessive radiation exposure can lead to health issues; specifically, spinal surgeons have been shown to be at an increased risk of thyroid cancer when exposed to excessive fluoroscopy times. ${ }^{[9]}$ Occupational radiation exposure has been linked to the development of cataracts, lymphoma, as well as solid tumors of the skin and breast. ${ }^{[17]}$

Fluoroscopy exposure during spinal surgery has been quantified by several studies. One study reported a mean fluoroscopy time per screw of 30 seconds, based on 1,438 screw placements (190 patients), with total radiation time during 
the procedure of approximately 5.7 minutes. ${ }^{[25]}$ Another study reported a mean minimally invasive spine procedure fluoroscopy time of 5.5 minutes with use of guidewires. ${ }^{[9]}$ Importantly, operating times, and thus fluoroscopy use, may be increased in cases where the guidewire has fractured or broken. A highly trained surgeon may be able to retrieve the displaced guidewire under endoscopic guidance which would require further fluoroscopy. ${ }^{[8]}$ Although the additional fluoroscopy is concerning for the patient, in cases of guidewireassociated complications, it is also a concern for the surgical staff who perform multiple minimally invasive spinal procedures per year. Although they wear protective equipment, the cumulative radiation exposure during surgery is problematic, especially for unprotected areas of the body (i.e. hands, face, etc.). ${ }^{[17]}$ A reduction in radiation exposure during such procedures would be beneficial, not just for the patient but also for the operating room staff and surgeon, mitigating potential health issues related to excessive radiation exposure.

\section{DisCUSSION AND CONCLUSIONS}

To date, there have been no published literature reviews that focus on the challenges associated with guidewire use in MIS of the spine. We therefore performed a narrative literature review that involved searching several key databases (e.g., PubMed and Embase), which resulted in 2,058 abstracts for review, of which 31 were relevant for inclusion. Despite the low number of included studies, a wide range of data could still be synthesized on various types of challenges, complications, and implications pertaining to guidewire use. Furthermore, this literature review calculated and reported guidewire complication rates; a particular strength of the review, as these rates are generally not explicitly reported in the literature.

This review demonstrates that the use of guidewires in MIS spinal procedures can be associated with substantial burdens and challenges. In some instances, major complications can arise from their use, including cerebrospinal fluid leak, infection, post-operative ileus and, in rare cases, death. Complication rates associated with guidewire use are not negligible, as they have been reported to be as high as $14.8 \%$. Even so, complications may still be under-reported, as most evidence is from retrospective, rather than prospective, observational studies. Guidewire use may also result in additional operative time, need for re-operation, or conversion to open surgery, potentially further increasing complication risk and associated healthcare resource use. Certain populations, such as those with obesity or osteoporosis, may be at higher risk of guidewire-related complications; as such, extra caution should be exercised if guidewires are to be used in these clinical circumstances. Finally, surgeon inexperience with guidewires may lead to an increased incidence of guidewire-associated complications. ${ }^{[13]}$ The ECRI Institute in the United States has estimated that approximately $70 \%$ of accidents involving medical devices can be attributed to user error or the technique used. ${ }^{[26]}$ As such, training programs for guidewire use in spinal surgery should be optimized to help prevent such complications and innovation in new product development should focus on minimizing the need for guidewires.

It is important to note that this literature review was not completely systematic given our objective was to be exploratory with the dearth of literature available, rather than have a very pre-defined and specific question. Although several databases and combinations of search terms were used, formal PRISMA guidelines were not applied in every circumstance (i.e., double abstract review with third reviewer assessing discrepancy, formal study quality assessment). It is therefore possible that some studies may have been missed. However, additional searches of a selection of relevant hospital trade journals produced minimal incremental data. Formal quality assessment of included studies was also not undertaken given the broad range of clinical studies that were included. Nevertheless, this review can perhaps be used as a framework for which further information can be systematically added as data become available.

This literature review is intended to raise awareness that important challenges can exist with guidewire use. Wellconducted prospective studies should be undertaken to shed more light on these findings. Furthermore, to optimize healthcare outcomes, techniques and innovative new product solutions should be developed and offered to help minimize complication risk, procedure inefficiencies, and radiation exposure associated with guidewire use in MIS of the spine.

\section{ACKNOWLEDGements}

The authors would like to acknowledge Becky Skidmore, who is an information specialist, for her contribution towards the literature search strategy for this review paper. We would also like to acknowledge Martin Walsh for his critical review of the manuscript.

\section{CONFlicts of InTERest Disclosure}

The authors declare they have no conflicts of interest. 


\section{REFERENCES}

[1] Onibokun A, Holly LT. Minimally invasive pedicle screw fixation. Operative Techniques in Neurosurgery. 2004; 7(2): 72-8. https: //doi.org/10.1053/j.otns.2004.08.003

[2] Wong AP, Smith ZA, Stadler JA, et al. Minimally Invasive Transforaminal Lumbar Interbody Fusion (MI-TLIF): Surgical technique, long-term 4-year prospective outcomes, and complications compared with an open TLIF cohort. Neurosurg Clin N Am. 2014; 25(2): 279304. PMid: 24703447. https : //doi.org/10.1016/j.nec. 2013 .12 .007

[3] Nasser R, Yadla S, Maltenfort MG, et al. Complications in spine surgery. J Neurosurg Spine. 2010; 13(2): 144-57. PMid: 20672949. https://doi.org/10.3171/2010.3.SPINE09369

[4] Mobbs RJ, Raley DA. Complications with K-wire insertion for percutaneous pedicle screws. J Spinal Disord Tech. 2014; 27(7): 3904. PMid: 23698104. https://doi.org/10.1097/BSD.0b013e 3182999380

[5] Guiroy A, Ciancio AM, Masanes NG, et al. Minimally invasive transforaminal lumbar fusion (MIS-TLIF): Initial results. Global Spine Journal. 2017; 2(Suppl 1): 292S.

[6] Guan X, Zhao S, Gu X, et al. Guide wire breakage during posterolateral endoscopic lumbar discectomy procedure: A case report. J Back Musculoskelet Rehabil. 2017; 30(2): 383-6. PMid: 27858672. https ://doi.org/10.3233/BMR-150295

[7] Scheer JK, Harvey MJ, Dahdaleh NS, et al. K-Wire fracture during minimally invasive transforaminal lumbar interbody fusion: Report of six cases and recommendations for avoidance and management. Surg Neurol Int. 2014; 5(Suppl 15): S520-2. PMid: 25593770. https : //doi.org/10.4103/2152-7806.148009

[8] Tchameni YTY, Morisod B, Simon C, et al. Endoscopic extraction of a prevertebral migrated guidewire after posterior cervical instrumentation. Spine Journal: Official Journal of the North American Spine Society. 2014; 14(8): 1800-1. PMid: 24667743. https://doi.org/10.1016/j.spinee.2014.03.033

[9] Agrawal S, Sucato DJ. The awl-staple versus guidewire method for placing vertebral screws in thoracoscopic anterior spinal fusion and instrumentation for adolescent idiopathic scoliosis. J Spinal Disord Tech. 2008; 21(6): 413-7. PMid: 18679096. https ://doi.org/10 .1097/BSD . 0b013e3181588261

[10] Lau D, Khan A, Terman SW, et al. Comparison of perioperative outcomes following open versus minimally invasive transforaminal lumbar interbody fusion in obese patients. Neurosurg Focus. 2013; 35(2): E10. PMid: 23905948. https ://doi.org/10.3171/2013 5.FOCUS13154

[11] Siddiqui SA, Singh PK, Garg K, et al. Intra-operative K-wire breakage during odontoid screw fixation. Neurol India. 2014; 62(2): 203-4. PMid: 24823739. https://doi.org/10.4103/0028-3886.13 2409

[12] Hafez A, Ibrahim TF, Raj R, et al. Delayed Migration of Fractured K-wire Causing Vertebral Artery Invagination After Anterior Atlantoaxial Fixation: A Case Report. World Neurosurg. 2016; 88: 695-10. PMid: 26748177. https://doi.org/10.1016/j.wneu .2015 .12 .082

[13] Guan X, Wu X, Fan G, et al. Endoscopic Retrieval of a Broken Guidewire During Spinal Surgery. Pain Physician. 2016; 19(2): E339E42. PMid: 26815261.
[14] Hao DJ, He BR, Wu QN, et al. Kirschner wire technique for pedicle screw insertion in thoracic scoliosis: Accuracy evaluation. Journal of Clinical Rehabilitative Tissue Engineering Research. 2008; 12(26): 5019-22.

[15] Thambiraj S, Quraishi NA. Intra-operative localisation of thoracic spine level: a simple “' $\mathrm{K}$ '-wire in pedicle” technique. Eur Spine J. 2012; 21(Suppl 2): S221-S4. PMid: 22349971. https : //doi.or $\mathrm{g} / 10.1007 / \mathrm{s} 00586-012-2193-3$

[16] Ringstrom MJ, Sullivan HG, Fundell LJ, et al. A new paradigm for staging pedicle screw-based spinal procedures: rationale, feasibility, safety, and efficacy. J Neurosurg Spine. 2007; 7(5): 521-32. PMid: 17977194. https://doi.org/10.3171/SPI-07/11/521

[17] Gebauer G, Anderson DG. Complications of Minimally Invasive Lumbar Spine Surgery. Semin Spine Surg. 2011; 23: 114-22. https://doi.org/10.1053/j.semss.2010.12.014

[18] Shim CS, Lee SH, Jung B, et al. Fluoroscopically assisted percutaneous translaminar facet screw fixation following anterior lumbar interbody fusion: technical report. Spine (Phila Pa 1976). 2005; 30(7): 838-43. https://doi.org/10.1097/01.brs.0000157 473.17313.6f

[19] Chung T, Thien C, Wang YY. A rare cause of postoperative paraplegia in minimally invasive spine surgery. Spine (Phila Pa 1976). 2014; 39(3): E228-E30. PMid: 24153167. https://doi.org/10.1097/ BRS. 0000000000000092

[20] Heini P, Scholl E, Wyler D, et al. Fatal cardiac tamponade associated with posterior spinal instrumentation: A case report. Spine. 1998; 23(20): 2226-30. PMid: 9802166. https://doi.org/10.1097/ 00007632-199810150-00017

[21] Barbagallo GM, Certo F, Visocchi M, et al. Multilevel mini-open TLIFs and percutaneous pedicle screw fixation: description of a simple technical nuance used to increase intraoperative safety and improve workflow. Tips and tricks and review of the literature. Neurosurg Rev. 2015; 38(2): 343-54. PMid: 25391632. https : //doi.org/10.1007/s10143-014-0589-8

[22] Gianaris TJ, Helbig GM, Horn EM. Percutaneous pedicle screw placement with computer-navigated mapping in place of Kirschner wires: clinical article. J Neurosurg Spine. 2013; 19(5): 608-13. PMid: 24010897. https://doi.org/10.3171/2013.7. SPINE121157

[23] Spitz SM, Sandhu FA, Voyadzis JM. Percutaneous "K-wireless" pedicle screw fixation technique: an evaluation of the initial experience of 100 screws with assessment of accuracy, radiation exposure, and procedure time. J Neurosurg Spine. 2015; 22(4): 422-31. PMid: 25658468. https : //doi.org/10.3171/2014.11. SPINE14181

[24] Santos ER, Sembrano JN, Yson SC, et al. Comparison of open and percutaneous lumbar pedicle screw revision rate using 3-D image guidance and intraoperative CT. Orthopedics. 2015; 38(2): e129e34. PMid: 25665118. https : //doi .org/10.3928/01477447-2 0150204-61

[25] Slawomir M. How to reduce interoperative fluoroscopy time in minimally invasive spine stabilization? Comparison of wireless and $\mathrm{K}$-Wire based screw insertion technique in fractures. Global Spine Journal. 2017; 2(Suppl 1): 296S.

[26] ECRI Institute. Dirty endoscopes top 2016 technology hazards list. OR Manager. 2016; 32(2): 21. 